\title{
Exposición a pesticidas y toxicidad reproductiva y del desarrollo en humanos. Análisis de la evidencia epidemiológica y experimental
}

\author{
María Fernanda Cavieres Fa. \\ Pesticide exposure and reproductive \\ and birth defects. Critical analysis of \\ epidemiological and experimental \\ evidence
}

Several epidemiological studies link pesticide exposure to reproductive and developmental toxicity. However, additional studies have shown little or no evidence to support such relationship. On the other hand, experimental data show that some pesticides may indeed alter the reproductive function or produce birth defects (as evidenced by intrauterine death, in utero growth retardation, visceral and skeletal malformations or functional deficits) in laboratory animals. This review is a critical analysis of the epidemiological and experimental evidence available to date, that links pesticide exposure with induction of reproductive or developmental defects. Factors that must be considered when establishing a cause-effect relationship are also discussed, including the shape of the dose-response curve, exposure to pesticides in chemical mixtures and the influence of genetic background (Rev Méd Chile 2004; 132: 873-9)

(Key-w ords: Abnormalities; Pesticides; Toxic actions)

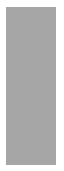

\begin{abstract}
Recibido el 9 de octubre, 2003. Aceptado en versión corregido el 19 de enero, 2004.
Facultad de Farmacia, Universidad de Valparaíso.

Trabajo financiado por Proyecto DIPUV/REG № 20/2002, Universidad de Valparaíso y Proyecto Davies Grant, Department of Zoology, Universidad de Wisconsin-Madison, Estados Unidos. aQuímico Farmaceútico.
\end{abstract}

\section{Magnitud del Problema}

En condiciones normales, una alta proporción de la población de todas las especies, incluyendo la humana, tiene un riesgo considerable de sufrir alteraciones reproductivas o del desarrollo, las que pueden manifestarse a través de condiciones

Correspondencia a: María Fernanda Cavieres F. Av Gran Bretaña 1093, Playa Ancha, Valparaíso. Fax: 32-508111.

E-mail: fernanda.cavieres@uv.cl tan variadas como infertilidad, aborto, malformaciones físicas o deficiencias funcionales a nivel neurológico, endocrino o inmune. En el caso específico de los defectos congénitos (malformaciones físicas o deficiencias funcionales), se ha estimado que $47 \%$ ocurre por causas desconocidas, $25 \%$ son genéticas, $25 \%$ son multifactoriales (es decir una combinación de factores genéticos y ambientales) y 3\% son causadas por agentes físicos, químicos o biológicos ${ }^{1}$. En este último caso, existe suficiente evidencia para afirmar que 
Tabla 1. Factores químicos, físicos o biológicos con evidencia suficiente para ser clasificados como teratógenos humanos (modificado de 1, 2, 18 y 19)

\begin{tabular}{|l}
\hline Factores físicos \\
Bomba atómica \\
Iodo radioactivo \\
Radioterapia \\
Rayos X \\
Factores biológicos \\
Citomegalovirus \\
Virus Herpes I y II \\
Śfilis \\
Virus de la Rubeola \\
Toxoplasmosis \\
Factores químicos \\
Desequilibrios metabólicos \\
Hiper e hipotiroidismo \\
Diabetes \\
Deficiencia de ácido fólico \\
Hipertermia \\
Fenilcetonuria \\
Enfermedad reumática \\
Sustancias de abuso \\
Anfetaminas \\
Cocaína \\
Etanol \\
LSD \\
Tolueno \\
Fármacos \\
Ácido valproico \\
Aminopterina \\
Andrógenos \\
Busulfan \\
Captopril \\
Cumarinas \\
Ciclofosfamida \\
Dietilestilbestrol \\
Difenilhidantoína \\
Enalapril \\
Etretinato \\
Ioduro \\
Isotretinoína \\
Litio \\
Metimazol \\
Penicilamina \\
Tetraciclinas \\
Talidomida \\
Trimetadiona \\
Contaminantes ambientales \\
Bifenilospoliclorados (PCBs) \\
Humo de cigarro \\
Mercurio orgánico \\
Plomo
\end{tabular}

entre 40 y 50 factores, incluyendo agentes químicos, infecciosos o físicos, ejercen toxicidad durante el desarrollo en humanos, (Tabla 1), un número muy bajo si se considera que poco más de 1.200 agentes teratogénicos han sido identificados en animales de experimentación ${ }^{1,2}$.

En el mundo, 5,4\% de los nacidos vivos presentan defectos congénitos, de estos 0,22\% corresponde a malformaciones ${ }^{3}$. En Chile, 1.060 personas $(1,2 \%)$ mueren cada año por defectos congénitos (malformaciones congénitas y anomalías cromosómicas), siendo aproximadamente 650 de ellos, niños menores de 1 año ${ }^{4}$. De acuerdo a Nazer y $\mathrm{col}^{5}$, las malformaciones congénitas han adquirido gran importancia como factores que afectan la morbilidad y mortalidad neonatal en Chile. De hecho, estos autores han calculado que la importancia relativa de las malformaciones congénitas como causa de mortalidad infantil aumentó desde $4,1 \%$ en 1970 a $35 \%$ en 1998, estimando, asimismo, que la tasa global de malformaciones aumenta anualmente en $0,06^{12}$ por 10.000 nacidos vivos. En este mismo estudio, se señala que las malformaciones relacionadas con el cierre del tubo neural, tienen una alta frecuencia en las regiones $\mathrm{V}$, VI y VIII, y se postula que factores ambientales, como la exposición a pesticidas, podrían estar influenciando estas estadísticas.

\section{EVIDENCIA EPIDEMIOLÓGICA}

El uso de sustancias agroquímicas es una práctica ampliamente generalizada en todo el mundo. El término pesticida, agrupa a un conjunto de compuestos con características químicas y actividades biológicas heterogéneas. Según su actividad, estos compuestos se clasifican en herbicidas, insecticidas, molusquicidas, fungicidas y rodenticidas, y por tanto, son útiles no sólo en la industria agrícola, donde se los emplea para proteger cultivos y aumentar rendimientos de cosechas, sino también a nivel doméstico, donde se los usa con frecuencia para eliminar insectos o roedores.

A pesar del gran uso de los pesticidas, se han publicado pocos informes que investiguen su toxicidad sobre la reproducción y la gestación en humanos, destacándose en Chile el trabajo reciente de Rojas y $\mathrm{col}^{6}$, el cual analiza estadísticamente la relación entre la incidencia de malformaciones 
congénitas y la exposición parental a pesticidas en recién nacidos en Rancagua.

Este estudio determinó que entre los años 1996 y 1998 hubo una prevalencia de 41,24\%o de malformaciones congénitas, destacándose las anomalías osteomusculares, del sistema nervioso central y cromosomopatías. Asimismo, 12,5\% de los mortinatos portaban malformaciones congénitas. Los autores encontraron una asociación positiva entre exposición a pesticidas e inducción de malformaciones congénitas, aún cuando destacan que es necesario hacer otros estudios para confirmar esta relación. A pesar de sus limitaciones, este estudio es el único que demuestra que la exposición a pesticidas podría estar influenciando la aparición de malformaciones congénitas en zonas de trabajo agrícola en Chile.

Estudios epidemiológicos en otras partes del mundo también relacionan la exposición a pesticidas con toxicidad del desarrollo en humanos. Pastore y $\mathrm{col}^{7}$, demostraron una clara asociación positiva entre la exposición ocupacional a pesticidas, especialmente durante la primera etapa del embarazo y el riesgo de mortinatalidad, en California, Estados Unidos. Kristensen y $\mathrm{col}^{8}$, asociaron defectos del sistema nervioso central y de las extremidades con el uso por padres y madres de equipos de aplicación de pesticidas en Noruega. Por otro lado, Bell y $\mathrm{col}^{9}$, demostraron que el riesgo de muerte fetal debido a anomalías congénitas, aumenta cuando existe exposición materna a pesticidas entre la $3^{\mathrm{a}}$ y $8^{\mathrm{a}}$ semana de embarazo.

Garry y col ${ }^{10,11}$ demostraron que la frecuencia de defectos del desarrollo en niños nacidos en zonas agrícolas de Minnesota, Estados Unidos, durante 1989-1991 era significativamente más alta que en niños nacidos en otras regiones de ese país. Adicionalmente, determinaron que los hijos de padres aplicadores de pesticidas tenían mayor riesgo de nacer con malformaciones y que la razón de sexo en los recién nacidos de esa zona podría estar siendo alterada por la exposición paterna a pesticidas, siendo herbicidas y fungicidas los que presentaron mayor asociación epidemiológica con la inducción de estos defectos. Estos estudios no controlan la exposición a pesticidas de las madres durante la gestación; sin embargo, ponen en evidencia que los defectos que aparecen en un recién nacido pueden no deberse a toxicidad del desarrollo (es decir pue- den no ocurrir por exposición materna) sino que por un defecto inducido a nivel de gameto masculino (por exposición paterna previa a la concepción).

Por otro lado, existen también estudios epidemiológicos que demuestran poca relación o una relación inexistente entre la exposición a pesticidas durante la gestación y la inducción de algún tipo de toxicidad del desarrollo. Así, un estudio caso-control realizado en Holanda determinó que había un efecto limitado de la exposición a pesticidas sobre la inducción de espina bífida en hijos de mujeres que realizaron trabajo agrícola durante el embarazo, aún cuando la contaminación del ambiente con pesticidas, independientemente del trabajo de la madre podría explicar el aumento del riesgo de inducción de espina bífi$\mathrm{da}^{12}$. Asimismo, Shaw y col ${ }^{13}$ tampoco demostraron una relación clara entre la exposición a pesticidas específicos y el riesgo de inducción de defectos del desarrollo mientras que Nurminen y $\mathrm{col}^{14}$ sólo determinaron una débil asociación entre exposición laboral materna a pesticidas y la presencia de malformaciones del sistema nervioso central y faciales y ninguna asociación con malformaciones esqueléticas.

En cuanto a toxicidad reproductiva, de Cock y $\mathrm{col}^{15}$, asociaron una disminución en la fertilidad (medida como un aumento en el número de ciclos menstruales sin anticonceptivos que demora una mujer en embarazarse), con la exposición a pesticidas, en mujeres holandesas esposas de agricultores frutícolas, aunque ningún grupo específico de pesticidas pudo identificarse como causante directo de tal efecto. Este mismo parámetro, empleado para estudiar fertilidad, fue utilizado en parejas canadienses por Curtis y $\mathrm{col}^{16}$, quienes demostraron que algunos pesticidas, como insecticidas organofosforados y herbicidas derivados del ácido fenoxiacético, estaban asociados con una disminución en la fecundidad de mujeres dedicadas a actividades laborales relacionadas con dichos pesticidas.

\section{EVIDENCIA EXPERIMENTAL}

Tal como se señala al inicio, unos 1.200 compuestos producen defectos congénitos en animales de experimentación, de los cuales un poco más de 40 
compuestos, señalados en la Tabla 1, son teratógenos humanos reconocidos $1,2,17,18$.

La Tabla 1 presenta un listado de los agentes físicos, biológicos y químicos para los cuales existen evidencias científicas suficientes para ser considerados teratógenos humanos. Como se ve, ninguna de las sustancias de esta lista es un pesticida.

Sin embargo, nadie puede desconocer que algunos pesticidas son efectivamente teratogénicos en animales de experimentación. Basta revisar la literatura para encontrar innumerables trabajos que demuestran este hecho (ver por ejemplo las monografías publicadas por la OMS con respecto a grupos específicos de pesticidas ${ }^{19,20}$, el texto de Kamrin ${ }^{21}$ o el capítulo dedicado a toxicología de pesticidas en Hayes $^{22}$ ).

La pregunta por qué, si los pesticidas causan malformaciones congénitas en animales de experimentación, no es posible determinar si las producen igualmente en humanos?, debe ser contestada con un criterio científico basado en, al menos, los siguientes fundamentos que subyacen a cualquier investigación toxicológica:

Factores que determinan una respuesta biológica. Desde un punto de vista estrictamente toxicológico, el establecimiento de una relación causaefecto directa e inequívoca entre exposición a pesticidas y toxicidad reproductiva o del desarrollo es extremadamente difícil, puesto que en una situación real existen numerosos factores confundentes que están ausentes en una investigación de laboratorio.

Relación dosis respuesta. El establecimiento de una relación dosis-respuesta es esencial para establecer la toxicidad reproductiva o del desarrollo de sustancias pesticidas en humanos ${ }^{23}$. El efecto que resulta de la interacción de una sustancia química con un sistema biológico sólo tiene sentido una vez establecida esta relación. En otras palabras, debe demostrarse claramente qué dosis de pesticida (o qué concentración ambiental de pesticida) induce un efecto tóxico específico. Desafortunadamente, para los pesticidas y muchos otros compuestos, sólo se conoce la relación dosis-respuesta en animales de experimentación, de tal forma que nos vemos en la necesidad de especular cuáles podrían ser los niveles tóxicos (y, por lo mismo, niveles seguros) de exposición a pesticidas.

Por otra parte, se sabe que la respuesta biológica es mayor a medida que aumenta la dosis (de acuerdo a Paracelso: «a dosis hace el veneno»). La curva dosis-respuesta de forma sigmoidea clásica refleja muy bien este postulado. Sin embargo, últimamente se está reconociendo que la respuesta biológica pudiera no ser lineal sino que en algunos casos puede seguir una forma de U, reflejando una inducción de respuesta a dosis bajas pero no a dosis muy altas. Este fenómeno se denomina hormesis y ha sido discutido recientemente por Calabresse y Baldwin ${ }^{24}$.

Desde el punto de vista de la toxicología reproductiva y del desarrollo de pesticidas, se hace necesario que los diseños experimentales incluyan dosis mucho más bajas que las consideradas hasta el momento, ya que al estudiar estas dosis aparecen efectos que de otra forma podrían pasar desapercibidos, los cuales son justamente las dosis y los efectos que dan a la relación la forma de una U. Además, las dosis más altas, tienen una relevancia toxicológica sin tener necesariamente una relevancia ambiental o real.

Exposición a mezclas químicas. Una de las limitaciones más importantes del conocimiento actual sobre toxicidad reproductiva y del desarrollo, es que la mayoría de los informes disponibles en la literatura indican resultados encontrados en un laboratorio en el que se han utilizado modelos animales expuestos a un pesticida único. La situación es distinta cuando se considera un escenario fuera del laboratorio, porque los humanos están expuestos a productos comerciales cuyas formulaciones pueden contener, no sólo mezclas de pesticidas con distinta actividad biológica, sino que también compuestos sin actividad pesticida, que se introducen al producto con el fin de mejorar su solubilidad o la estabilidad del producto ${ }^{25}$.

Las interacciones entre compuestos activos e inactivos pueden aumentar o disminuir un efecto tóxico, mientras que existe también la posibilidad de que los efectos de un producto comercial sobre sistemas biológicos sean debidos sólo a los componentes inactivos. Por ejemplo, estudios recientes con el herbicida glifosato 26,27 , han permitido postular que la interacción entre pesticidas y componentes inactivos puede llevar a una 
exacerbación de los efectos tóxicos producto quizás de que ciertos ingredientes, como detergentes, pueden mejorar la biodisponibilidad del pesticida para las células animales.

Otro punto a considerar es que todas las personas viven en distintas condiciones fisiológicas y de alimentación, ingesta de medicamentos o exposición a otros xenobióticos ambientales, todas sustancias químicas que pueden también interactuar con el pesticida. Evidentemente, la exposición simultánea a múltiples sustancias químicas es una situación que debe ser estudiada.

Susceptibilidad individual. Cada vez se hace más evidente que los seres humanos (así como todos los organismos vivos, muchos de los cuales son utilizados como modelos experimentales) responden en forma distinta a un mismo compuesto e incluso a una misma dosis de él. La explicación a este fenómeno se encuentra en la variabilidad genética que existe entre un individuo y otro, lo cual puede manifestarse, por ejemplo, en la expresión o la falta de expresión de distintas formas de proteínas receptoras, transportadoras 0 metabolizadoras de pesticidas. Lamentablemente, no existe todavía un conocimiento profundo de las diferentes expresiones de proteínas en distintas etnias. Por lo mismo, la predicción de que un pesticida inducirá un problema reproductivo 0 algún defecto del desarrollo en la población chilena, basado en estudios epidemiológicos desarrollados en otras partes de mundo, debe hacerse considerando este aspecto.

Indudablemente, la identificación de genes responsables de una mayor o menor vulnerabilidad a la toxicidad reproductiva o del desarrollo ayudará también a dilucidar el riesgo de inducción de estos tipos de toxicidad.

Otros factores. James Wilson, considerado el padre de la teratología moderna, señaló en 1973, los seis principios que rigen la inducción de un defecto del desarrollo ${ }^{28}$. Algunos de estos principios, como la susceptibilidad individual y la relación dosis-respuesta, han sido comentados en los párrafos anteriores. Al discutir el tema del difícil esclarecimiento de la relación causa-efecto entre pesticidas e inducción de toxicidad, vale la pena destacar al menos otros dos principios de Wilson:
Uno de estos principios, por todos conocido, es que la susceptibilidad a la toxicidad varía a medida que la gestación avanza. Para establecer fehacientemente la relación causa-efecto debemos ser capaces de estudiar exhaustivamente cuándo ocurre la exposición al pesticida durante un embarazo.

El otro principio tiene relación con las manifestaciones de la toxicidad del desarmollo. Generalmente, se asume que sólo las malformaciones congénitas, el retraso en el crecimiento intrauterino y la muerte embrionaria o fetal son consecuencias de la toxicidad del desarrollo. Sin embargo, defectos funcionales pueden también ser el resultado de un proceso teratogénico y en este respecto existen evidencias de que los pesticidas pueden estar induciendo toxicidad neurológica ${ }^{29,30}$, endocrina ${ }^{31,32}$ e inmune ${ }^{33}$ en niños expuestos in utero a pesticidas.

\section{CONCLUSIÓN}

Shepard 17,34 ha sugerido que para que un factor físico, biológico o químico pueda ser considerado teratógeno humano se deben considerar una amalgama de resultados de estudios epidemiológicos así como experimentales (Tabla 2). De igual forma, la Food and Drug Administration de Estados Unidos (FDA), ha establecido criterios para la clasificación de los medicamentos según su potencial teratogénico, los que también se basan en una sólida evidencia epidemiológica y experimental.

Sever ${ }^{35}$ publicó en 1994, una completa revisión sobre malformaciones congénitas y riesgos reproductivos en relación a la exposición ocupacional de padres y madres concluyendo que a esa fecha no había evidencia convincente de que exista tal relación. Casi diez años después, todavía se puede asegurar lo mismo.

Sin embargo, el hecho de que no podamos demostrar científicamente que la relación exista, no significa que esta no existe. El establecimiento de la relación causa-efecto para los cientos de compuestos químicos utilizados como pesticidas es una tarea difícil, aunque no imposible. En nuestra experiencia ${ }^{36,37}$, la evidencia científica se mejora notoriamente al considerar, en los diseños experimentales, los factores mencionados anteriormente. De esta forma la situación experimental se asemeja mucho más a la situación real, facilitando así el proceso de extrapolación de datos. 
Tabla 2. Criterios sugeridos para la determinación de teratogénos humanos (modificado de 14 y 31).

Criterios de Shepard ${ }^{1}$

1. Existe evidencia contundente de exposición intrauterina al factor en una etapa crítica del desarrollo.

2. Existen datos concordantes obtenidos en dos o más estudios epidemiológicos de alta calidad (estudios en los cuales se controlen factores confundentes, con un tamaño de población adecuado, se excluyan factor de sesgo positivo o negativo, que sean en lo posible prospectivos y con un riesgo relativo establecido en seis o más).

3. Existe una clara descripción de los casos clínicos. La existencia de un defecto o síndrome específico complementa esta información.

4. Si la exposición ambiental es poco frecuente, entonces la aparición del defecto también es infrecuente.

5. La demostración de teratogenicidad en animales de experimentación es importante pero no esencial.

6. La asociación exposición-efecto debe tener un sentido biológico.

7. Existe evidencia experimental que la sustancia actúa como tal y no como un metabolito.

Criterio de la FDA para medicamentos teratógenos

Categoría A: Estudios controlados en mujeres embarazadas no demuestran riesgo para el feto en el primer trimestre y la probabilidad de daño fetal es remota.

Categoría B: Estudios en animales no han demostrado riesgo para el feto pero no existen estudios controlados en mujeres embarazadas o estudios en animales han demostrado efectos adversos que no han sido confirmados en humanos.

Categoría C: Estudios en animales han demostrado efectos adversos pero no hay estudios en humanos o no hay estudios ni en animales ni en humanos.

Categoría D: Hay evidencia de riesgo fetal en humanos, pero su uso puede ser aceptado a pesar de este riesgo, «según la relación riesgo/beneficio que se establezca».

Categoría X: Estudios en animales y seres humanos han demostrado que la sustancia es teratogénica y el riesgo de su uso claramente sobrepasa los beneficios.

\section{REFERENCIAS}

1. NRC, National Research Council. Scientific Frontiers in Developmental Toxicology and Risk Assessment. National Academy Press, Washington, 2000.

2. FinNel RH, Gelineau-Van Waes J, Eudy JD, RosenQUIST TH. Molecular basis of environmentally induced birth defects. Annu Rev Pharmacol Toxicol 2002; 42: 181-208.

3. OMS, Organización Mundial de la Salud. Primary health care approaches for prevention an control of congenital and genetic disorders. Report of a WHO meeting, Cairo, 1999.

4. MINSAL, Ministerio de Salud de Chile. Estadísticas de Natalidad y Mortalidad. Departamento de Estadísticas e Información de Salud, 1999.

5. Nazer JH, Aravena T, Cifuentes L Malformaciones congénitas en Chile. Un problema emergente (período 1995-1999). Rev Méd Chile 2001; 129: 895-904.

6. Rojas A, OJeda ME, Barraza X. Malformaciones congénitas y exposición a pesticidas. Rev Méd Chile 2000; 128: 399-404.

7. Pastore LM, Hertz-Picciotto I, Beaumont JJ. Risk of still birth from occupational and residential exposures. Occup Environ Med 1997; 54: 511-8.

8. Kristensen P, Irgens LM, Andersen A, Snewigen Bye A, SundheIm L. Birth defects among offspring of Norwegian farmers, 1967-1991. Epidemiology, 1997; 8: 537-44.

9. Bell EM, Hertz-Picciotto I, Beaumont JJ. A casecontrol study of pesticides and fetal death due to congenital anomalies. Epidemiology 2001; 12: 148-56.

10. GaRRY VF, SCHREINEMACHERS D, HARKINS ME, GRIFFTH J. Pesticide Appliers, biocides and birth defects in rural Minnesota. Environ Health Perspect 1996; 104: 394-9. 
11. GarRY VF, Harkins ME, ERICKSON LL, LONG-Simpson LK, HoLANd SE, Burroughs BL. Birth defects, season of conception, and sex of children born to pesticide applicators living in the Red River Valley of Minnesota, USA. Environ Health Perspect 2002; 110(S3): 441-9.

12. Blatter BM, Roeleveld N, Zielhuis GA, Gabreels FJM, VeRBEek ALM. Maternal occupational exposure during pregnancy and the risk of spina bifida. Occup Environ Med 1996; 53: 80-6.

13. Shaw GM, Wasserman CR, O'maley CD, Neison V, JACKSON RJ. Maternal pesticide exposure from multiple sources and selected congenital anomalies. Epidemiology 1999; 10: 60-6.

14. Nurminen T, Rantala K, Kurppa K, Holmberg PC. Agricultural work during pregnancy and selected structural malformations in Finland. Epidemiology 1995; 6: 23-30.

15. De Cock J, Westveer K, Heederick D, Te Velde E, VAN Kooij R. Time to pregnancy and occupational exposure to pesticides in fruit growers in The Netherlands. Occup Environ Med 1994; 51: 693-9.

16. Curtis KM, Savitz Da, Weinberg CR, Arbuckie TE. The effect of pesticide exposure on time to pregnancy. Epidemiology 1999; 10: 112-7.

17. SHEPARD TH. Agents that cause birth defects. Yonsei Med J 1995; 36: 394-7.

18. Rogers JM, KavLock RJ. Developmental Toxicology. En: Klassen CD, ed. Casarett \& Doull's Toxicology. The Basic Science of Poisons. 5th ed. New York: McGraw Hill, 1996; 301-31.

19. OMS. Organización Mundial de la Salud. Organophosphorus insecticides: a general introduction. Environmental Health Criteria 63, Ginebra, 1986.

20. OMS. Organización Mundial de la Salud. 2,4Dichlorophenoxyacetic acid. Environmental Health Criteria 29, Ginebra, 1980.

21. KamRin MA. Pesticide Profiles. Toxicity, Environmental Impact and Fate. Boca Raton: CRC Lewis Publishers, 1997.

22. Stevens JT, Breckenridge CB. Crop Protection Chemicals. En: Hayes WA (ed). Principles and Methods of Toxicology. Fourth ed., Philadelphia: Taylor \& Francis, 2001, 565-648.

23. SHEPARD TH. Dose-response in human teratology. Teratology 2002; 65: 199-205.

24. Calabresse EJ, Baldwin LA. Hormesis: the doseresponse revolution. Ann rev Pharmacol Toxicol 2003; 43: 175-97.
25. Tominack RL. Herbicide Formulations. Clinical Toxicol 2000; 38: 129-35.

26. Walsh LP, McCormick C, Martin C, Stocco DM. Roundup inhibits steroidogenesis by disrupting steroidogenic acute regulatory (StAR) protein expression. Environ Health Perspect 2000; 108: 769-76.

27. Marc J, Mulner-LoriLon O, Boulben S, Hureau D, Durand G, Belue R. Pesticide Roundup provokes cell division dysfunction at the level of CDK1/ cyclin B activation. Chem Res Toxicol 2002; 32631.

28. WiLSON JG. Environment and birth defects. New York: Academic Press, 1973.

29. Guilete EA, Meza MM, Aquilar MG, Soto AD, GARCIA IE. An anthropological approach to the evaluation of preschool children exposed to pesticides in Mexico. Environ Health Perspect 1998; 106: 347-53.

30. TIISON HA. Developmental neurotoxicology of endocrine disruptors and pesticides: identification of information gaps and research needs. Environ Health Perspect 1998; 106: 807-11.

31. Colborn T, Vom SaAl FS, Soto AM. Developmental effects of endocrine-disrupting chemicals in wildlife and humans. Environ Health Perspect, 1993; 101: 378-84.

32. Bigsby R, Chapin RE, Daston GP, Davis BJ, Gorski J, Gray LE, Howdeshell KL, Zoeler RT, Vom SaAl FS. Evaluating the effects of endocrine function during development. Environ Health Perspect 1999; 107: 613-8.

33. Holladay SD, Smalowicz RJ. Development of the murine and human immune system: differential effects of immunotoxicants depend on time of exposure. Environ Health Perspect 2000; 108: 463-73.

34. Shepard TH. Annual commentary on human teratogens. Teratology 2002; 66: 275-7.

35. SEVER LE. Congenital malformations related to occupational reproductive hazards. Occ Med St Art Rev 1994; 9: 471-95.

36. Cavieres MF, Jaeger J, Porter W. Developmental toxicity of a commercial herbicide product in mice. I. Effects on embryo implantation and litter size. Environ Health Perspect 2002; 110: 1081-5.

37. CAVIERES MF, SMIth SM. Genetic and developmental modulation of cardiac deficits in prenatal alcohol exposure. Alcoholism: Clin Exp Res 2000; 24: 102-9. 